\title{
Sentinel Lymph Node Procedure Leads to Upstaging of Patients with Resectable Colon Cancer: Results of the Swiss Prospective, Multicenter Study Sentinel Lymph Node Procedure in Colon Cancer
}

\author{
Carsten T. Viehl, MD ${ }^{1}$, Ulrich Guller, MD, $\mathrm{MHS}^{2}$, Ramona Cecini, $\mathrm{MD}^{3}$, Igor Langer, $\mathrm{MD}^{4}$, Alex Ochsner, $\mathrm{MD}^{5}$, \\ Luigi Terracciano, $\mathrm{MD}^{6}$, Hans-Martin Riehle, $\mathrm{MD}^{7}$, Urban Laffer, MD, FRCS ${ }^{3}$, Daniel Oertli, MD, FACS, FRCS ${ }^{1}$, \\ and Markus Zuber, MD $^{5}$
}

${ }^{1}$ Department of Surgery, University of Basel, Basel, Switzerland; ${ }^{2}$ Division of Medical Oncology, Cantonal Hospital St. Gallen, St. Gallen, Switzerland; ${ }^{3}$ Department of Surgery, Hospital Center Biel, Biel, Switzerland; ${ }^{4}$ Department of Surgery, Lindenhof Hospital, Berne, Switzerland; ${ }^{5}$ Department of Surgery, Cantonal Hospital Olten, Olten, Switzerland; ${ }^{6}$ Institute of Pathology, University of Basel, Basel, Switzerland; ${ }^{7}$ Viollier Histopathology/Cytology, Basel, Switzerland

\begin{abstract}
Background. The value of the sentinel lymph node (SLN) procedure in colon cancer patients remains a matter of debate. The objective of this prospective, multicenter trial was 3-fold: to determine the identification rate and accuracy of the SLN procedure in patients with resectable colon cancer; to evaluate the learning curve of the SLN procedure; and to assess the extent of upstaging due to the SLN procedure.

Methods. One hundred seventy-four consecutive colon cancer patients were enrolled onto this prospective trial. They underwent an intraoperative SLN procedure with isosulfan blue $1 \%$ injected peritumorally followed by open standard colon resection with oncologic lymphadenectomy. Three levels of each SLN were stained with hematoxylin and eosin (H\&E) and immunostained with the pancytokeratin marker AE1/AE3 if $\mathrm{H} \& \mathrm{E}$ was negative.
\end{abstract}

The first two authors contributed equally to this study.

Presented in part at the Society of Surgical Oncology's 61st Annual Cancer Symposium, Chicago, IL, March 13-16, 2008, and the Annual Meeting of the Swiss Society of Surgery, Basel, Switzerland, June 28-30, 2008.

(C) Society of Surgical Oncology 2012

First Received: 30 August 2011;

Published Online: 10 February 2012

M. Zuber, MD

e-mail: markus.zuber@spital.so.ch
Results. SLN identification rate and accuracy were $89.1 \%$ and $83.9 \%$, respectively. SLN were significantly more likely to contain tumor infiltrates than non-SLN $(P<0.001)$. Both SLN identification rate $(P=0.021)$ and the sensitivity of the procedure $(P=0.043)$ significantly improved with experience. The use of immunohistochemistry in SLN resulted in an upstaging of $15.4 \%$ (16 of 104) stage I and II patients considered node-negative in initial H\&E analysis.

Conclusions. The SLN procedure for colon cancer has good identification and accuracy rates, which further improve with increasing experience. Most importantly, the SLN procedure results in upstaging of $>15 \%$ of nodenegative patients. The potential advantage of performing the SLN procedure appears to be particularly important in these patients because they may potentially benefit from adjuvant therapy.

The sentinel lymph node (SLN) principle assumes that the lymphatic spread of a given cancer occurs in an orderly centrifugal pattern. ${ }^{1}$ Therefore, first-tier lymph nodes have a greater probability of harboring nodal tumor infiltrates than second or third tier lymph nodes. First-tier lymph nodes are considered to be the sentinel nodes and therefore to reflect whether the regional lymph nodes harbor tumor cells. The identification of one or a few SLNs greatly facilitates nodal staging in some malignancies (e.g., breast cancer or malignant melanoma) because the analysis of a smaller number of lymph nodes is required. Moreover, in 
search of small nodal tumor infiltrates, SLN can be analyzed more thoroughly, including serial sectioning and the use of immunohistochemistry (IHC). To perform such in-depth analyses for all resected lymph nodes would be prohibitively expensive and time-consuming.

The SLN procedure has gained widespread acceptance for axillary staging of breast cancer patients. ${ }^{2}$ It is now well known that the SLN procedure leads to reduced morbidity, improved staging, and better outcomes in breast cancer patients. ${ }^{3,4}$ However, unlike breast cancer, the SLN procedure for colon cancer patients is still under investigation and remains a matter of debate. Only a few multicenter trials, including one randomized, controlled study, evaluating the procedure have been published so far. ${ }^{5-8}$ The goal of performing the SLN procedure in colon cancer patients is to improve the accurate nodal staging and to facilitate identification of small nodal tumor infiltrates $(\leq 2 \mathrm{~mm})$ in a time- and cost-effective way. This is particularly important in patients considered to be node-negative after conventional nodal staging-that is, stage I and II disease according to American Joint Committee on Cancer (AJCC) criteria. ${ }^{9}$ These patients have a potential risk of being understaged because a relevant fraction of patients harbor nodal metastases that can be found with careful reexamination of the resected lymph nodes. ${ }^{10-12}$ Therefore, hypothetically, the potential benefits of adjuvant therapy on disease-free and overall survival are withheld from patients whose disease is understaged. This risk is particularly important in patients with a low number of reported lymph nodes-for example, fewer than 12 lymph nodes. ${ }^{13-15}$ The SLN procedure has been suggested to improve accurate nodal staging in colon cancer patients. ${ }^{16}$

The objective of this prospective, multicenter trial was 3 -fold: to determine the identification rate and accuracy of the SLN procedure for consecutive colon cancer patients; to analyze the learning curve for the SLN procedure in colon cancer patients; and most importantly, to assess the extent of upstaging due to the SLN procedure.

\section{METHODS}

\section{Study Settings}

This study is a prospective multicenter trial with a standardized protocol ("Swiss Prospective, Multicenter Study Sentinel Lymph Node Procedure in Colon Cancer"). Three Swiss academic and university-affiliated institutions enrolled patients from May 2000 through December 2006. The study was approved by all ethical committees of the participating centers and was conducted in compliance with the institutional guidelines for experimental investigation with human subjects as well as according to the
Declaration of Helsinki. All patients provided written informed consent. The trial has been registered at ClinicalTrials.gov (NCT00826579).

\section{Patients}

Patients with biopsy-proven resectable colon cancer, AJCC stage I-III, scheduled for open surgery were eligible. $^{9}$ Exclusion criteria were distant metastases, rectal cancers, prior abdominal cancer surgery, history of other malignancies, allergy to isosulfan blue, pregnancy, and breast-feeding. In total, 203 patients were considered for inclusion in this study (Fig. 1). Twenty-nine patients (14.3\%) did not meet the eligibility criteria and were thus excluded. We therefore report on 174 eligible and evaluable patients. Patient characteristics are listed in Table 1.

\section{SLN Procedure}

The technique of the SLN procedure was standardized in the protocol and followed the recommendations described in detail by Saha et al. as well as by our group. ${ }^{16-18}$ Briefly, after careful mobilization of the affected colon segment, isosulfan blue $1 \%$ (median $2.0 \mathrm{ml}$, range $0.2-10.0 \mathrm{ml}$; Lymphazurin $1 \%$, Ben Venue Labs, Bedford, $\mathrm{OH}$; or isosulfan blue USZ 1\%, University Hospital of Zurich, Zurich, Switzerland) was injected in vivo into the subserosa circumferentially around the tumor with a tuberculin syringe with 29-gauge needle. The amount of blue dye to be injected was not specified in the protocol. However, on the basis of our own data, surgeons tended to inject higher volumes of blue dye for larger tumors in the second part of the study. ${ }^{18}$ Lymph nodes in the mesentery staining blue during the first 10 min were marked as SLN with a suture. Only these marked nodes were considered SLN; additional blue nodes detected later by the pathologist were not considered SLN. The procedure was followed by a resection of the affected colon segment with standard oncologic lymphadenectomy.

\section{Histopathologic Examination}

All marked SLN were processed separately as standardized in the study protocol: Irrespective of lymph node size, five serial sections of each SLN were obtained at 3 different representative levels. The first section of each level was stained with hematoxylin and eosin (H\&E). If no metastatic deposits were detectable by $\mathrm{H} \& \mathrm{E}$, the fourth section of each level was immunostained with the pancytokeratin marker AE1/AE3 (DakoCytomation, Glostrup, Denmark). Manual dissection of the fixed surgical specimens was performed to identify the remaining lymph nodes (non-SLN). The mesentery was sliced at $0.3-0.5 \mathrm{~cm}$ 


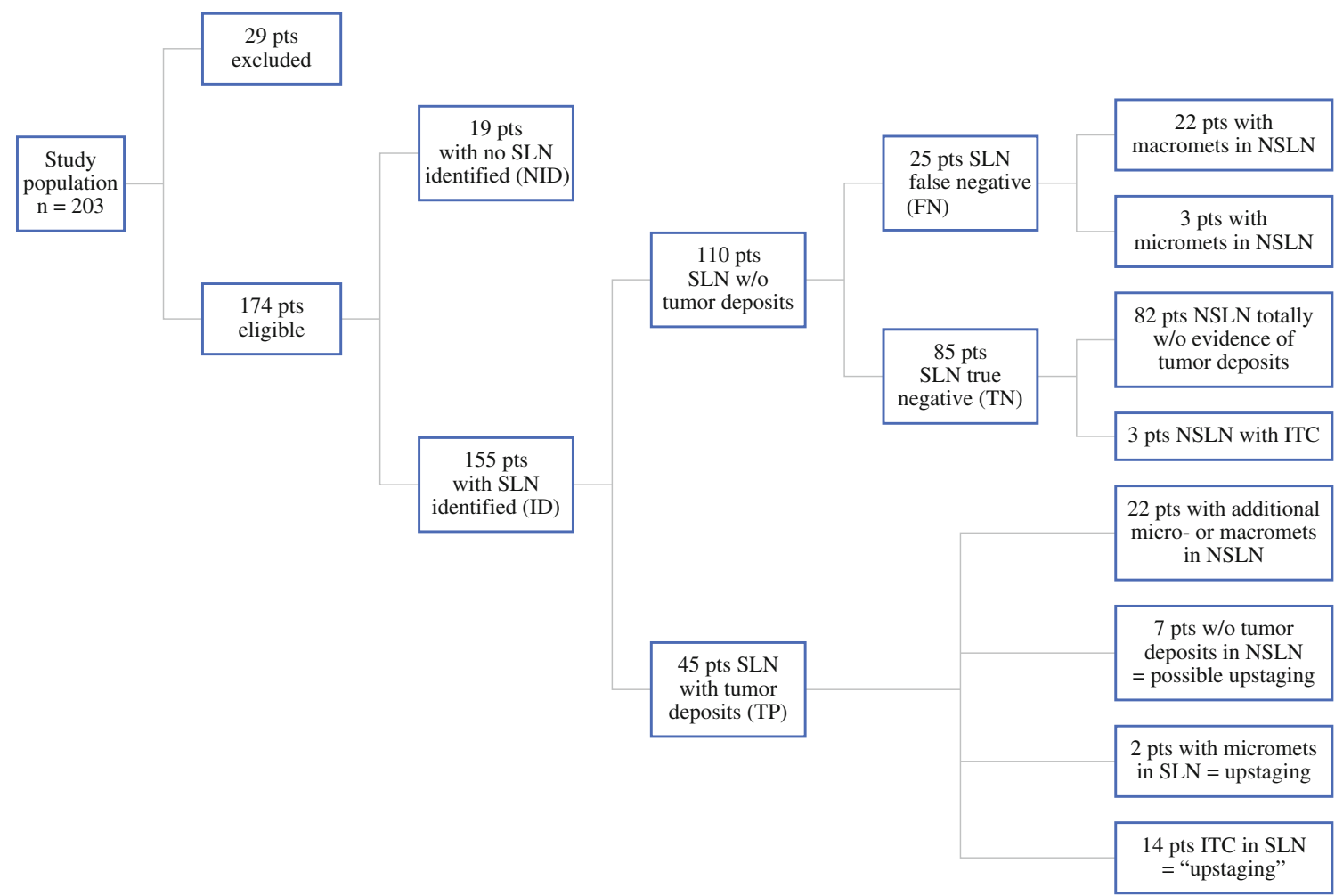

FIG. 1 Study flow chart. $F N$ false-negative group, ID identification group, ITC isolated tumor cell, mets metastases, NID nonidentification group, $N S L N$ non-sentinel lymph node, pts patients, $S L N$ sentinel lymph node, $T N$ true-negative group, $T P$ true-positive group, $w / o$ without

TABLE 1 Characteristics of the population

\begin{tabular}{ll}
\hline Characteristic & Value \\
\hline Total & $174(100 \%)$ \\
Gender & \\
Male & $95(54.6 \%)$ \\
Female & $79(45.4 \%)$ \\
Age, y, median (range) & $74(27-93)$ \\
AJCC stage & \\
I & $32(18.4 \%)$ \\
II & $78(44.8 \%)$ \\
III & $64(36.8 \%)$ \\
Localization of primary tumor & \\
Right hemicolon & $75(43.1 \%)$ \\
Left hemicolon & $31(17.8 \%)$ \\
Sigmoid colon & $68(39.1 \%)$ \\
Center & \\
Center 1 & $109(62.6 \%)$ \\
Center 2 & $38(21.8 \%)$ \\
Center 3 & $27(15.5 \%)$ \\
No. of surgeons & \\
Center 1 & 7 \\
Center 2 & 13 \\
Center 3 & 7 \\
\hline AJCC Amerin Joint \\
\end{tabular}

AJCC American Joint Committee on Cancer ${ }^{9}$ intervals to allow thorough visual and digital inspection. Done carefully this gross dissection method will reveal all lymph nodes $0.3 \mathrm{~cm}$ in size and larger. The non-SLN were bivalved and examined by H\&E. If no macrometastases were found in the SLN or in the non-SLN, all non-SLN were examined equally to the SLN with step sections and IHC. All cytokeratin positive cells were confirmed to be tumor cells by microscopic reevaluation of the immunostained sections after counterstaining with hemalaun.

\section{Staging}

Staging was performed according to the 6th edition of the AJCC staging manual - that is, lymph nodes with micrometastases were considered positive, whereas lymph nodes with isolated tumor cells were considered negative. ${ }^{9}$ The definitions of tumor cell infiltrates according to the 6th edition of the AJCC staging manual were as follows: macrometastases $>2.0 \mathrm{~mm}$, micrometastases $>0.2$ to $\leq 2.0 \mathrm{~mm}$, and isolated tumor cells (ITC) $\leq 0.2 \mathrm{~mm} .{ }^{9,19}$ In the present study, micrometastases and ITC were subsumed as small nodal tumor infiltrates. Patients considered node-negative after initial $\mathrm{H} \& \mathrm{E}$ analysis, but in whom small nodal tumor infiltrates were detected after in-depth analyses (serial sections and IHC), were considered upstaged - that is, to 
pN1(mi) (stage III) if micrometastases were detected, or to pNO(i+) if ITC were found.

\section{Statistical Analysis}

For descriptive statistics, median values with ranges are given. Categorical variables were analyzed with the Fisher's exact test. For continuous variables, the Student's $t$-test was used. Possible correlations were analyzed with the two-sided Pearson correlation. Accuracy ([true-positive + true-negative cases]/all cases), sensitivity, specificity, negative and positive predictive values, and false-negative and falsepositive rates were calculated as described by Walter. ${ }^{20}$ To assess the learning curve of the SLN procedure, patients from each center were assigned to groups according to their date of surgery; the first 20 patients from each center built one group, the second 20 patients from each center another, and so forth. Subsequently, SLN identification rate and sensitivity of the procedure were calculated for each group, and compared by Fisher's exact test. Statistical analyses were performed with SPSS 13.0 for Macintosh (SPSS, Chicago, IL). A $P$ value of $<0.05$ was considered to be significant. All $P$ values were two-sided.

\section{RESULTS}

In the present prospective multicenter study, 174 eligible and evaluable patients were enrolled. The median age was 73.7 years with a range from 27.3 to 93.0 years. There was a light male preponderance $(54.6 \%)$. The characteristics of the patient sample are given in Table 1.

\section{Identification Rate and Accuracy}

At least one SLN was identified in 155 of 174 eligible patients (identification rate 89.1\%; Table 2). In 130 of these 155 patients the SLN results corresponded to the nonSLN results - that is, the SLNs were either true-positive or true-negative for the detection of macrometastasis (accuracy of the SLN procedure 83.9\%; Table 2). However, in 25 patients $(16.1 \%)$, SLN showed no macro- or micrometastases while non-SLN did (false-negative SLN result). Additional rates describing the SLN procedure are given in Table 2.

\section{Technical Details of the SLN Procedure and Characteristics of the Nodal Staging}

In 155 patients with SLN identification, the median injected volume of isosulfan blue $1 \%$ was $2.0 \mathrm{ml}$ $(0.2-10.0 \mathrm{ml})$. Time to detection of the first SLN ranged from 0.5 to $22 \mathrm{~min}$, with a median of $5 \mathrm{~min}$. A median of 5
TABLE 2 Results of the SLN procedure

\begin{tabular}{llr}
\hline Result & $n / N$ & $\%$ \\
\hline Identification rate & $155 / 174$ & 89.1 \\
Accuracy & $130 / 155$ & 83.9 \\
Sensitivity & $31 / 56$ & 55.4 \\
False-negative rate & $25 / 56$ & 44.6 \\
Specificity & $99 / 99$ & 100.0 \\
False-positive rate & $0 / 99$ & 0.0 \\
Negative predictive value & $99 / 124$ & 79.8 \\
Positive predictive value & $31 / 31$ & 100.0 \\
\hline
\end{tabular}

SLN (1-10) were tagged intraoperatively. There was no correlation between the volume of isosulfan blue injected and the number of SLN detected $(P=0.288)$. In seven patients $(4.5 \%)$, a single macrometastasis was found in the SLN. The median number of all lymph nodes analyzed (SLN and non-SLN) in this study was 24 (5-62). Only five patients (3.2\%) had fewer than the minimum required number of 12 lymph nodes described in the pathology report. ${ }^{15}$ In total, 4000 lymph nodes were analyzed. Of those, $562(14.1 \%)$ were SLN, and 3438 (85.9\%) were non-SLN. Forty-nine $(8.7 \%)$ of 562 SLN were found to have nodal metastases by H\&E, and $31(5.5 \%)$ of 562 by IHC. Of 3438 non-SLN, 149 ( $4.3 \%)$ were positive by H\&E and $11(0.3 \%)$ of 3438 by IHC. Therefore, SLN were significantly more likely to harbor nodal tumor infiltrates compared to non-SLN, based on both diagnosis by H\&E $(P<0.001)$ and IHC $(P<0.001)$. No SLN procedure related complications (e.g., allergic reactions) were reported in any of the patients.

\section{Upstaging}

To assess a potential upstaging due to the SLN procedure, we analyzed 104 patients who were node-negative in initial H\&E analysis at three levels (stage I and II patients according to conventional analysis). In 16 of these patients (15.4\%), small nodal tumor infiltrates (micrometastases or isolated tumor cells) were found in the SLN by multilevelsectioning and the use of IHC (Table 3 ). These patients were upstaged to $\mathrm{pN} 1(\mathrm{mi})$ or $\mathrm{pN} 0(\mathrm{i}+)$, respectively. ${ }^{9}$ In six additional patients $(5.8 \%)$, small nodal tumor infiltrates were found in non-SLN only. However, this upstaging cannot be attributed to the SLN procedure because these additional small nodal tumor infiltrates were found in the non-SLN but not in the SLN.

\section{Learning Curve}

The learning curve was calculated by patients' groups of 20 each, according to their date of surgery as described in the method section (Table 4). SLN identification rate 
TABLE 3 Detection of small tumor infiltrates in sentinel lymph nodes of node-negative patients in initial $\mathrm{H} \& \mathrm{E}$ analysis (patients with stage I and II disease)

\begin{tabular}{llcc}
\hline Stage & Micrometastases, $n$ & Isolated tumor cells, $n$ & Total \\
\hline I & 2 & 1 & $3 / 31(9.7 \%)$ \\
II & - & 13 & $13 / 73(17.8 \%)$ \\
Total & 2 & 14 & $16 / 104(15.4 \%)$ \\
\hline
\end{tabular}

Patients with micrometastases were upstaged to stage III according to the 6th edition of the American Joint Committee on Cancer staging manual ${ }^{9}$; patients with isolated tumor cells were "upstaged" from pNO to $\mathrm{pNO}(\mathrm{i}+)$

TABLE 4 Learning curve of the SLN procedure

\begin{tabular}{lll}
\hline No. of cases & SLN identification rate & Sensitivity \\
\hline $1-20$ & $80.0 \%$ & $42.9 \%$ \\
$21-40$ & $88.9 \%$ & $68.4 \%$ \\
$41-60$ & $95.0 \%$ & $60.0 \%$ \\
$\leq 61$ & $98.0 \%$ & $85.0 \%$ \\
$P$ & 0.021 & 0.043
\end{tabular}

Patients from each center were assigned to groups according to their date of surgery; the first 20 patients from each center built one group (cases 1-20), the second 20 patients another (cases 21-40), and so forth. It is noteworthy that only center 1 enrolled more than 40 patients onto the study; therefore, "cases $41-60$ " and "cases $\geq 61$ " represent the learning curve of a single center

significantly improved with center experience $(P=0.021)$, as did the sensitivity of the SLN procedure $(P=0.043)$ with a SLN identification rate of $98 \%$ and a sensitivity of $85 \%$ if the center experience exceeds 60 procedures.

\section{DISCUSSION}

The findings of the present Swiss prospective, multicenter study provide compelling evidence that the in vivo SLN procedure for colon cancer has good identification and accuracy rates. Most importantly, the SLN procedure results in upstaging of $>15 \%$ of node-negative patients. The potential advantage of performing the SLN procedure appears to be particularly important in node-negative patients as they may benefit from adjuvant therapy.

With a median of 24 analyzed lymph nodes per patient, nodal staging was excellent in our study. Only five patients (3.2\%) had fewer than the minimal required 12 lymph nodes assessed. ${ }^{15}$ These favorable lymph node counts could be caused in part by trial awareness by the participating surgeons and pathologists as well as the fact that blue staining was used. However, a posthoc analysis at one of the participating centers (center 1) did not show a significant change in lymph node yields for patients operated on for colon cancer before and after the initiation of the study (data not shown). Our data compare favorably with findings from the Surveillance, Epidemiology and End Results cancer registry, where a median of only nine lymph nodes were identified. ${ }^{21}$ Moreover, the number of retrieved lymph nodes in the present investigation clearly exceeds the lymph node harvest from the randomized, controlled COST trial, in which 872 colon cancer patients were randomized to open versus laparoscopic colon cancer resection and only 12 lymph nodes were found both in the open and laparoscopic group. ${ }^{22}$ The lymph node harvest in our investigation represents an important quality indicator of properly performed surgical oncologic resections as well as meticulous pathologic analysis in our consecutive sample of colon cancer patients. An excellent nodal staging leaves little room for improvement; nonetheless, $>15 \%$ of stage I and II patients were upstaged with the SLN procedure in the present investigation. This is the most relevant finding of our prospective, multicenter study. The upstaging of stage I and II colon cancer patients was even higher in the Dutch and the German multicenter trials. ${ }^{7,8}$ In a recently published systematic review, a mean upstaging of $15 \%$ of node-negative patients after conventional histopathologic analysis was found. ${ }^{14}$ Patients who are upstaged as a result of the SLN procedure may constitute a population at higher risk of recurrence within the stage I and II subset. ${ }^{14}$ Hypothetically, these patients might benefit from adjuvant therapy. However, while this issue has not yet been proven, it is currently under investigation in a Dutch randomized, controlled trial (En Route Trial, NCT01097265). ${ }^{23}$

American Society of Clinical Oncology and National Comprehensive Cancer Network guidelines now recommend adjuvant chemotherapy to be considered in high-risk stage II patients with one or several of the following features: pT4 tumor, poor differentiation of the primary tumor, fewer than 12 lymph nodes analyzed, presence of lymphovascular invasion, localized perforation, and bowel obstruction. $^{15,24}$ Although $13(17.8 \%)$ of 73 stage II patients in our study showed small tumor infiltrates in the SLN, only $5(38.5 \%)$ of 13 of these patients had features that now prompt the consideration of adjuvant chemotherapy; all other patients ( 8 of $13,61.5 \%$ ) with small nodal tumor infiltrates would have missed out on this possibly beneficial treatment.

Moreover, seven patients (4.5\%) in our study had only a single macrometastasis in the SLN, a situation referred to as "possible upstaging." Because the lymph nodes containing the single metastasis might be missed by the pathologist if only a few lymph nodes are analyzed, the SLN procedure possibly contributed to the correct nodal staging in these patients as well.

Some oncologists have stated concerns about the safety of the SLN procedure, particularly regarding the in vivo injection of isosulfan blue. However, in the present study, 
no SLN procedure-related complications were observed. Therefore, we provide compelling evidence that the performance of the intraoperative SLN procedure in colon cancer patients is not harmful.

The results of the present investigation underline the important learning curve for the in vivo SLN procedure in colon cancer patients. Both SLN identification as well as the sensitivity significantly increased with higher center experience. In fact, if more than 60 procedures were performed, the identification rate was close to $100 \%$ with a sensitivity of $85 \%$. It is thus crucial that a surgeon with expertise in the SLN procedure supervises a beginner to achieve good results and obtain maximal benefits. Alternatively, a dedicated person might perform an ex vivo SLN procedure after the resection of the affected colon segment. However, because we performed exclusively the in vivo SLN procedure in this study, we cannot compare the two methods. Moreover, despite a good identification rate at the end of the learning curve with only $15 \%$ false-negative SLN, this false-negative rate is still too high to exclusively identify and analyze SLN. Thus, this procedure certainly leads to a better staging in colon cancer patients; however, the performance of a more limited colon cancer resection that is based on the SLN remains clearly investigational.

Surprisingly, identification rates as well as the sensitivity of the SLN procedure are lower in the German study and in the present multicenter trial compared to some investigations from the United States. ${ }^{6,7,25}$ Indeed, Bembenek et al. (identification rate $85 \%$, sensitivity $54 \%$ ) reported similar results to our study (identification rate $89 \%$, sensitivity $55.4 \%){ }^{7}$ The identification rate in the investigations from Bilchik et al. and Saha et al. are close to $100 \%$ with a sensitivity of approximately $90 \%$. The reason for this phenomenon-a European versus a U.S. pattern-remains to be elucidated. ${ }^{6,25}$

It is interesting that the ratio of micrometastases versus isolated tumor cells (ITC) is clearly lower in this prospective trial compared to the German multicenter study or a large investigation from the United States. ${ }^{7,10}$ The reason for this observation remains unclear. Regardless, colon cancer patients undergoing a SLN procedure who are found to be negative even after ultrastaging (serial sectioning of SLN and use of IHC in addition to standard H\&E) represent a subset of patients with excellent prognosis. ${ }^{10}$ Through a more thorough staging, the SLN procedure allows for a more accurate prognostic classification of stage I and II colon cancer patients (e.g., truly node-negative patients). Therefore, performing the SLN procedure in colon cancer patients has both therapeutic and prognostic implications.

In conclusion, the SLN procedure for colon cancer has good identification and accuracy rates, which further improve with increasing experience. Most importantly, the
SLN procedure results in upstaging of $>15 \%$ of patients with stage I and II disease. The potential advantage of performing the SLN procedure appears to be particularly important in these patients because they may benefit from adjuvant chemotherapy.

\section{REFERENCES}

1. Morton DL, Wen DR, Wong JH, et al. Technical details of intraoperative lymphatic mapping for early stage melanoma. Arch Surg. 1992;127:392-9.

2. Giuliano AE, Kirgan DM, Guenther JM, Morton DL. Lymphatic mapping and sentinel lymphadenectomy for breast cancer. Ann Surg. 1994;220:391-8.

3. Langer I, Guller U, Hsu-Schmitz SF, et al. Sentinel lymph node biopsy is associated with improved survival compared to level I and II axillary lymph node dissection in node negative breast cancer patients. Eur J Surg Oncol. 2009;35:805-13.

4. Langer I, Guller U, Berclaz G, et al. Morbidity of sentinel lymph node biopsy (SLN) alone versus SLN and completion axillary lymph node dissection after breast cancer surgery: a prospective Swiss multicenter study on 659 patients. Ann Surg. 2007;245: 452-61.

5. Stojadinovic A, Nissan A, Protic M, et al. Prospective randomized study comparing sentinel lymph node evaluation with standard pathologic evaluation for the staging of colon carcinoma: results from the United States Military Cancer Institute Clinical Trials Group Study GI-01. Ann Surg. 2007;245:846-57.

6. Saha S, Sehgal R, Patel M, et al. A multicenter trial of sentinel lymph node mapping in colorectal cancer: prognostic implications for nodal staging and recurrence. Am J Surg. 2006;191: 305-10.

7. Bembenek AE, Rosenberg R, Wagler E, et al. Sentinel lymph node biopsy in colon cancer: a prospective multicenter trial. Ann Surg. 2007;245:858-63.

8. Kelder W, Braat AE, Karrenbeld A, et al. The sentinel node procedure in colon carcinoma: a multi-centre study in The Netherlands. Int J Colorectal Dis. 2007;22:1509-14.

9. Greene FL. The American Joint Committee on Cancer: updating the strategies in cancer staging. Bull Am Coll Surg. 2002;87:13-5.

10. Bilchik A, Nissan A, Wainberg Z, et al. Surgical quality and nodal ultrastaging is associated with long-term disease-free survival in early colorectal cancer: an analysis of 2 international multicenter prospective trials. Ann Surg. 2010;252:467-74.

11. Faerden AE, Sjo OH, Bukholm IR, et al. Lymph node micrometastases and isolated tumor cells influence survival in stage I and II colon cancer. Dis Colon Rectum. 2011;54:200-6.

12. Belt EJ, van Stijn MF, Bril $\mathrm{H}$, et al. Lymph node negative colorectal cancers with isolated tumor deposits should be classified and treated as stage III. Ann Surg Oncol. 2010;17:3203-11.

13. Swanson RS, Compton CC, Stewart AK, Bland KI. The prognosis of T3N0 colon cancer is dependent on the number of lymph nodes examined. Ann Surg Oncol. 2003;10:65-71.

14. van der Pas MH, Meijer S, Hoekstra OS, et al. Sentinel-lymphnode procedure in colon and rectal cancer: a systematic review and meta-analysis. Lancet Oncol. 2011;12:540-50.

15. Guidelines: colon cancer. In: National Comprehensive Cancer Network (NCCN); 2011. p. COL-3. http://www.ncen.org.

16. Viehl CT, Guller U, Hamel CT, et al. Carbon dye staining of sentinel lymph nodes facilitates microstaging of colon cancer patients. World J Surg. 2006;30:453-6.

17. Saha S, Wiese D, Badin J, et al. Technical details of sentinel lymph node mapping in colorectal cancer and its impact on staging. Ann Surg Oncol. 2000;7:120-4. 
18. Viehl CT, Hamel CT, Marti WR, et al. Identification of sentinel lymph nodes in colon cancer depends on the amount of dye injected relative to tumor size. World J Surg. 2003;27:1285-90.

19. Hermanek P, Hutter RV, Sobin LH, Wittekind C; International Union Against Cancer. Classification of isolated tumor cells and micrometastasis. Cancer. 1999;86:2668-73.

20. Walter SD. Sensitivity. In: Armitage P, Colton T, editors. Encyclopedia of biostatistics. Chichester: Wiley; 1998. p. 405354.

21. Chen SL, Bilchik AJ. More extensive nodal dissection improves survival for stages I to III of colon cancer: a population-based study. Ann Surg. 2006;244:602-10.

22. A comparison of laparoscopically assisted and open colectomy for colon cancer. N Engl J Med. 2004;350:2050-9.
23. Study of micrometastases in patients with stage I or stage II localized colon cancer that can be removed by surgery. Trial NCT01097265. ClinicalTrials.gov. http://www.clinicaltrials.gov/ ct $2 /$ show/NCT01097265?term $=$ nct01097265\&rank=1.

24. Benson AB III, Schrag D, Somerfield MR, et al. American Society of Clinical Oncology recommendations on adjuvant chemotherapy for stage II colon cancer. J Clin Oncol. 2004;22: 3408-19.

25. Bilchik AJ, DiNome M, Saha S, et al. Prospective multicenter trial of staging adequacy in colon cancer: preliminary results. Arch Surg. 2006;141:527-33. 